\title{
$\mathrm{Ni}\left(\mathrm{NO}_{3}\right)_{2}$ 浓度对 $\mathrm{Ni} /$ 还原氧化石墨微观结构 及微波吸收性能的影响
}

\author{
祝 海, 杨丽, 刘洪波, 陈惠, 夏笑虹 \\ (湖南大学 材料科学与工程学院, 长沙 410082)
}

\begin{abstract}
摘 要: 以 $\mathrm{Ni}\left(\mathrm{NO}_{3}\right)_{2}$ 为 $\mathrm{Ni}$ 源, 利用液相浸渍法在氧化石墨层间吸附 $\mathrm{Ni}^{2+}$, 通过 $\mathrm{H}_{2}$ 热还原制备出 $\mathrm{Ni}$ /还原氧化石墨纳 米复合材料。采用扫描电子显微镜(SEM)、X 射线衍射仪(XRD)及网络矢量分析仪等对样品的结构及性能进行分析 和表征, 研究了 $\mathrm{Ni}\left(\mathrm{NO}_{3}\right)_{2}$ 浓度对材料微观形貌及电磁性能的影响。结果表明, 所制备材料为纳米级 $\mathrm{Ni}$ 颗粒与 $\mathrm{RGO}$ 的复合体，具有优良的微波吸收性能; 当 $\mathrm{Ni}\left(\mathrm{NO}_{3}\right)_{2}$ 浓度为 $1.5 \mathrm{~mol} / \mathrm{L}$ 时，材料电磁吸收性能最佳，在 $2 \sim 18 \mathrm{GHz}$ 频率 范围内, 材料厚度为 $2 \mathrm{~mm}$ 时, 反射损耗 $(R L)$ 小于 $-5 \mathrm{~dB}$ 的频率范围可达 $9 \mathrm{GHz}, R L_{\max }$ 可达 $-40 \mathrm{~dB}$ 。

关 键 词: $\mathrm{Ni}$; 氧化石墨; 纳米复合材料; 微波吸收

中图分类号: TB333 文献标识码: A
\end{abstract}

\section{Effect of Nickel Nitrate Concentration on Microstructure and Microwave Absorbing Property of Ni/RGO Nanocomposite Material}

\author{
ZHU Hai, YANG Li, LIU Hong-Bo, CHEN Hui, XIA Xiao-Hong \\ (College of Material Science and Engineering, Hunan University, Changsha 410082, China)
}

\begin{abstract}
A technique for obtaining reduced graphite oxide (RGO) with Ni nanoparticle dispersed on the surface via chemical vapor deposition (CVD) method was reported. XRD and SEM observations were carried out to confirm the microstructure of Ni/RGO composite. Magnetic property of Ni/RGO was characterized by vector network analyzer. The influence of $\mathrm{Ni}\left(\mathrm{NO}_{3}\right)_{2}$ concentration on micromorphology and microwave absorbing property were also discussed. As the concentration of $\mathrm{Ni}\left(\mathrm{NO}_{3}\right)_{2}$ is $1.5 \mathrm{~mol} / \mathrm{L}$, the range of the reflection loss $(R L)$ less than $-5 \mathrm{~dB}$ is $9 \mathrm{GHz}$, of which the max value reaches $-40 \mathrm{~dB}$.
\end{abstract}

Key words: nickel; graphite oxide; nanocomposite material; microwave absorbing

随着电子科技的迅速发展, 越来越多的电子设 备进入到人们的生活当中，而使用这些电子设备的 同时，所伴随的电磁波辐射不仅干扰电子设备的正 常工作，也会影响人体健康 ${ }^{[1-3]}$, 采用吸波材料是减 轻电磁波辐射的有效方式之一。以铁氧体为代表的 过渡金属磁性材料目前已广泛用作微波吸收材料 ${ }^{[4-6]}$, 但是其比重大、吸收频段窄、吸收率低 ${ }^{[7-8]}$ 等缺点 使其不能满足先进吸波材料 “轻、薄、强、宽” 的
要求，限制了其应用范围。

$\mathrm{Ni}$ 金属与传统铁氧体相比，具有较高的居里点 和饱和磁化强度, 并且在高频下仍能保持较高相对 复磁导率, 这些独特的优点使 $\mathrm{Ni}$ 基材料开始引起更 多微波吸收领域内学者的关注。 $\mathrm{Wu}$ 等 ${ }^{[9]}$ 利用水合肼 和有机酸还原制备了不同尺寸的单质 $\mathrm{Ni}$ 。研究发现 当 $\mathrm{Ni}$ 粒径在 $100 \mathrm{~nm}$ 时, 在 $9.3 \mathrm{GHz}$ 处其最大理论 反射损耗 $\left(R L_{\text {max }}\right)$ 可达 $-26.4 \mathrm{~dB} ;$ Zhao 等 ${ }^{[10]}$ 则利用电 
弧放电法制备出具有核壳结构的 $\mathrm{Ni} / \mathrm{C}$ 纳米复合材 料，该方法可将 $\mathrm{Ni}$ 颗粒尺寸控制在 $100 \mathrm{~nm}$ 以内。 以 $20 \mathrm{~nm}$ 的 $\mathrm{Ni} / \mathrm{C}$ 颗粒制成厚度为 $3.5 \mathrm{~mm}$ 的吸波材 料, $R L_{\max }$ 可达 $-34 \mathrm{~dB}$ 。以上研究结果表明, 较小的 $\mathrm{Ni}$ 颗粒尺寸有利于材料的吸波性能的改善。然而纯 的纳米金属容易氧化、团聚, 且容易自燃, 难以储存 和运输, 而核壳结构的制备方法成本高昂, 流程复 杂, 不易量产, 大大限制了其应用。

氧化石墨(Graphite Oxide, GO)是一种二维层状 材料, 该材料是石墨经过强氧化处理, 在层间插入 大量含氧基团所形成的石墨层间化合物。其在结构 上保留了石墨的层状结构, 而且由于层间含有大量 如羧基、羟基等含氧极性基团, 使其具有良好的亲 水性和离子吸附性能, 这些特点使得氧化石墨易与 多种材料复合 ${ }^{[11-14]}$ 。作者所在课题组是较早开展此 类研究的团队之一, 已成功制备了 $\mathrm{Fe} / \mathrm{RGO}$ 和 $\mathrm{FeCo} / \mathrm{RGO}$ 等体系的纳米复合材料 ${ }^{[15-16]}$, 并获得了 较好的微波吸收效果。但至今尚未见采用这一途径 探索合成 $\mathrm{Ni} /$ 还原氧化石墨纳米复合材料, 以获得 更好的微波吸收性能。

为了开发性能更优的微波吸收材料, 本研究采 用液相浸渍法将 $\mathrm{Ni}^{2+}$ 吸附到氧化石墨层间, 在 $\mathrm{H}_{2}$ 气 氛下加热还原制备出 $\mathrm{Ni}$ / 还原氧化石墨 (简称 $\mathrm{Ni} / \mathrm{RGO}$ )纳米复合材料, 考察了 $\mathrm{Ni}\left(\mathrm{NO}_{3}\right)_{2}$ 浓度对材 料微观形貌、电磁参数以及微波吸收性能的影响。

\section{1 实验方法}

\section{1 氧化石墨 GO 的制备}

采用 Hummers 法制备氧化石墨, 在干燥的烧杯 中加入 $690 \mathrm{~mL}$ 浓度为 $98 \%$ 的浓硫酸, 用低温冷却 液循环䡕冷却至 $4^{\circ} \mathrm{C}$ 以下, 边摚拌边将 $30 \mathrm{~g}$ 天然鳞 片石墨和 $15 \mathrm{~g} \mathrm{NaNO}_{3}$ 混合物加入到烧杯中, 然后再 缓慢加入 $90 \mathrm{~g} \mathrm{KMnO}_{4}$, 过程中控制混合物温度在 $20^{\circ} \mathrm{C}$ 以下, 完成低温反应。将烧杯移入 $35^{\circ} \mathrm{C}$ 的恒温 水浴中, 持续摚拌 $30 \mathrm{~min}$, 完成中温反应。最后用梨 形分液漏斗向烧杯中滴加 $1400 \mathrm{~mL}$ 去离子水, 保持缓 慢升温, 总温度控制在 $98^{\circ} \mathrm{C}$ 以下, 随后将烧杯转入 $140^{\circ} \mathrm{C}$ 油浴中搅拌 $15 \mathrm{~min}$, 加入 $220 \mathrm{~mL} 5 \%$ 的 $\mathrm{H}_{2} \mathrm{O}_{2}$, 得到金黄色产物。趁热过滤, 用 $5 \mathrm{wt} \%$ 的 $\mathrm{HCl}$ 和蒸馏 水充分洗涤直至滤液中无 $\mathrm{SO}_{4}{ }^{2-}$ 离子。利用透析袋将产 物洗至中性, 冷冻干燥, 得到氧化石墨 $(\mathrm{GO})$ 粉末。

\section{2 镍/还原氧化石墨纳米复合材料的制备}

配制一定浓度的 $\mathrm{Ni}\left(\mathrm{NO}_{3}\right)_{2}$ 溶液, 向溶液中加入 一定质量氧化石墨, 摚拌均匀后进行超声分散和真
空浸渍各 $1 \mathrm{~h}$, 然后对物料进行抽滤处理。取出滤饼 并在 $60^{\circ} \mathrm{C}$ 下干燥, 研磨后即得到 $\mathrm{Ni}\left(\mathrm{NO}_{3}\right)_{2} / \mathrm{GO}$ 复合 物。由于 $\mathrm{GO}$ 在快速加热至 $140 \sim 210^{\circ} \mathrm{C}$ 时, 会急剧 膨胀爆炸, 所以在 $\mathrm{H}_{2}$ 还原之前需对 $\mathrm{Ni}\left(\mathrm{NO}_{3}\right)_{2} / \mathrm{GO}$ 复 合物预先进行低温慢速热处理。然后在 $650^{\circ} \mathrm{C}$ 的 $\mathrm{H}_{2}$ 气氛下还原 $4 \mathrm{~h}$, 得到 $\mathrm{Ni} / \mathrm{RGO}$ 纳米复合材料。根据 制备复合材料时 $\mathrm{Ni}\left(\mathrm{NO}_{3}\right)_{2}$ 浓度的不同，将样品分别 标记为 $\mathrm{Ni} / \mathrm{RGO}-0.5 、 \mathrm{Ni} / \mathrm{RGO}-1$ 、 Ni/RGO-1.5、 $\mathrm{Ni} / \mathrm{RGO}-2\left(0.5\right.$ 即表示 $\mathrm{Ni}\left(\mathrm{NO}_{3}\right)_{2}$ 浓度为 $0.5 \mathrm{~mol} / \mathrm{L}$, 依 此类推)。

\section{3 样品性能与表征}

样品的微观表面形貌由 Hitachi S-4800 型扫描 电子显微镜观察。采用 Simens D-5000 型全自动 X 射线衍射仪分析 $\mathrm{Ni} / \mathrm{RGO}$ 纳米复合材料的晶体结构, 扫描范围为 $5^{\circ} \sim 80^{\circ}$, 扫描速度为 $5^{\circ} / \mathrm{min}$ 。利用所制 得样品为吸波剂, 以 $m$ (样品) $/ m$ (石蜡 $)=1 / 1$ 的比例制 备同轴检测环, 采用 Agilent 8720ET 网络矢量分析 仪在 $2 \sim 18 \mathrm{GHz}$ 范围内测定电磁参数, 根据测定的 电磁参数计算试样在 2 18 GHz 范围内的理论反射 损耗。

\section{2 结果与讨论}

\section{$2.1 \mathrm{Ni}\left(\mathrm{NO}_{3}\right)_{2}$ 浓度对 $\mathrm{Ni} / \mathrm{RGO}$ 纳米复合材料微 观结构的影响}

图 1 为 $\mathrm{Ni}\left(\mathrm{NO}_{3}\right)_{2}$ 浓度分别为 $0.5 、 1 、 1.5$ 和 $2.0 \mathrm{~mol} / \mathrm{L}$ 时制得的 $\mathrm{Ni} / \mathrm{RGO}$ 纳米复合材料的 SEM 照 片, 其中图 1(c)右上角为样品的 $\operatorname{EDS}$ 能谱图。由 EDS 结果可知, 还原之后的样品主要由 $\mathrm{Ni} 、 \mathrm{C}$ 及少 量 $\mathrm{O}$ 和 $\mathrm{S}$ 元素构成。结合 XRD 结果可知, 少量 $\mathrm{O}$ 和 $\mathrm{S}$ 元素主要来自于样品中少量未完全还原的 $\mathrm{GO}$ 。 SEM 结果显示, 四个样品的表面均覆盖大量的颗粒, 其主要成分为金属 $\mathrm{Ni}$ 单质, 其形成原理如下: 在液 相真空浸渍过程中，通过氧化石墨片层上的羧基、 羟基等含氧极性基团的吸附作用, $\mathrm{Ni}^{2+}$ 在分子水平 上均匀地吸附在氧化石墨层间, 随后在 $\mathrm{H}_{2}$ 加热条件 下，氧化石墨因层间的含氧基团受热分解成气体逸 出或被 $\mathrm{H}_{2}$ 还原而转化为还原氧化石墨，而 $\mathrm{Ni}^{2+}$ 亦被 还原成 0 价 $\mathrm{Ni}$ 单质, 与此同时, 刚生成的细小 $\mathrm{Ni}$ 单质颗粒会继续生长、融并, 最终形成图中所示颗 粒形貌 ${ }^{[17]}$ 。随着 $\mathrm{Ni}\left(\mathrm{NO}_{3}\right)_{2}$ 浓度的增大, 颗粒在还原 氧化石墨(RGO)表面的分布密度逐渐升高，颗粒尺 寸也呈增大趋势。样品 Ni/RGO-0.5(图 1(a))的颗粒 尺寸约为 5 7 $\mathrm{nm}$, 均匀散布在 RGO 片层表面及层 间，但存在尚未完全覆盖的区域。当 $\mathrm{Ni}\left(\mathrm{NO}_{3}\right)_{2}$ 浓度 

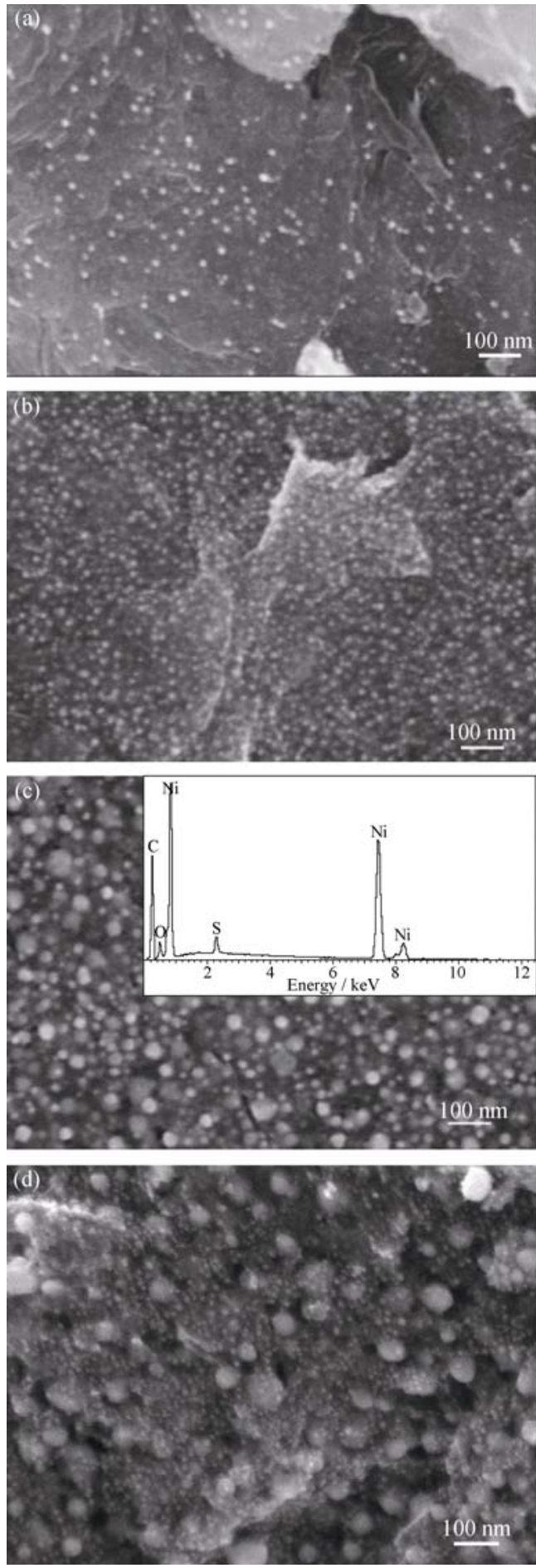

图 1 Ni/RGO-0.5(a) 、 Ni/RGO-1.0(b) Ni/RGO-1.5(c) 与 $\mathrm{Ni} / \mathrm{RGO}-2.0(\mathrm{~d})$ 样品的 SEM 照片及 EDS 图谱

Fig. 1 SEM images of Ni/RGO-0.5 (a), Ni/RGO-1.0 (b), $\mathrm{Ni} / \mathrm{RGO}-1.5$ with EDS pattern in inset (c), and Ni/RGO-2.0 (d)

增加到 $1.0 \mathrm{~mol} / \mathrm{L}$ (图 1(b))时, 颗粒尺寸增至 $10 \mathrm{~nm}$ 左右, 颗粒大小均匀且片层基本被 $\mathrm{Ni}$ 颗粒所覆盖, 说明随着 $\mathrm{Ni}\left(\mathrm{NO}_{3}\right)_{2}$ 浓度的增加, 吸附在氧化石墨片 层中的 $\mathrm{Ni}$ 金属离子数量也随之增加, 所以在相同的 还原条件下, 由于有更加充足的 $\mathrm{Ni}$ 离子, $\mathrm{Ni} / \mathrm{RGO}-1$ 所生成的 $\mathrm{Ni}$ 颗粒尺寸较大, 分布密度也更高。而当
浓度进一步增大到 $1.5 \mathrm{~mol} / \mathrm{L}$ (图 1(c))时，颗粒尺寸 明显增大, 且呈不匀化。而浓度为 $2.0 \mathrm{~mol} / \mathrm{L}$ (图 1(d)) 时，Ni 颗粒呈现两种不同粒径分布，小颗粒直径大 约在 $10 \mathrm{~nm}$ 左右, 而大颗粒直径可达 $50 \mathrm{~nm}$ 。造成 该现象的原因可能是由于 $\mathrm{Ni}$ 离子浓度过大，使得局 部分布不均, 吸附 $\mathrm{Ni}$ 离子浓度较高的区域在还原过 程中融并形成较大的颗粒。

图 2 为不同 $\mathrm{Ni}\left(\mathrm{NO}_{3}\right)_{2}$ 浓度下制得的 $\mathrm{Ni} / \mathrm{RGO}$ 纳 米复合材料的 XRD 图谱。不难发现，四个样品的 XRD 图谱中皆出现了石墨在 $26^{\circ}$ 左右的特征峰, 该峰的存在说明样品中的氧化石墨经过 $\mathrm{H}_{2}$ 热还原 处理后，层间的含氧基团有所去除，同时，该特征 峰并不十分尖锐, 说明样品经过还原处理后, 其中 的石墨相堆垛结构受到了一定程度的破坏，但总体 上仍维持片层结构状态 ${ }^{[18-19]}$ 。 Ni/RGO-0.5 样品中出 现了单质 $\mathrm{Ni}$ 的(111)和(200)晶面衍射特征峰, 其峰 强较弱且不尖锐。利用谢乐公式可计算出 $\mathrm{Ni}$ 晶粒的 平均尺寸为 $5.8 \mathrm{~nm}$, 与 $\mathrm{SEM}$ 观察结果基本一致，说 明 $\mathrm{Ni}\left(\mathrm{NO}_{3}\right)_{2}$ 浓度较低时形成的 $\mathrm{Ni}$ 单质数量较少, 晶 体结构也存在较多缺陷。随着 $\mathrm{Ni}\left(\mathrm{NO}_{3}\right)_{2}$ 浓度升高至 $1 \mathrm{~mol} / \mathrm{L}, \mathrm{Ni} / \mathrm{RGO}-1.0$ 中 $\mathrm{Ni}$ 相的衍射强度明显提高, XRD 图谱中出现了 $\mathrm{Ni}(220)$ 晶面衍射峰, 而石墨 (002)衍射峰的相对强度有所减弱, $\mathrm{Ni}$ 成为该样品 的主相, 其晶粒尺寸也增大至约 $12.1 \mathrm{~nm}$ 。随着 $\mathrm{Ni}\left(\mathrm{NO}_{3}\right)_{2}$ 浓度的升高, $\mathrm{Ni}$ 颗粒尺寸不断增大，其特征 衍射峰的相对强度也随之增强。当浓度升至 $2.0 \mathrm{~mol} / \mathrm{L}$ 时, 石墨的特征峰已变得非常微弱。

为了进一步了解 $\mathrm{Ni} / \mathrm{RGO}$ 复合材料中官能团的 消除，选用原始氧化石墨以及 Ni/RGO-1.0 样品进行 FTIR 红外分析(图 3)。由图 3 可知, 代表氧化石墨

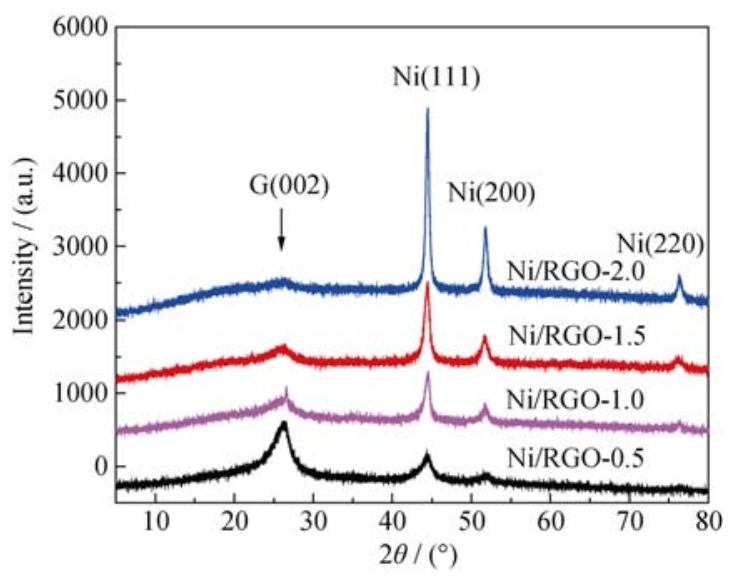

图 $2 \mathrm{Ni} / \mathrm{RGO}-0.5 、 \mathrm{Ni} / \mathrm{RGO}-1.0 、 \mathrm{Ni} / \mathrm{RGO}-1.5$ 与 $\mathrm{Ni} / \mathrm{RGO}-2.0$ 样品的 XRD 图谱

Fig. 2 XRD patterns of Ni/RGO-0.5, Ni/RGO-1.0, Ni/RGO-1.5, and $\mathrm{Ni} / \mathrm{RGO}-2.0$ 


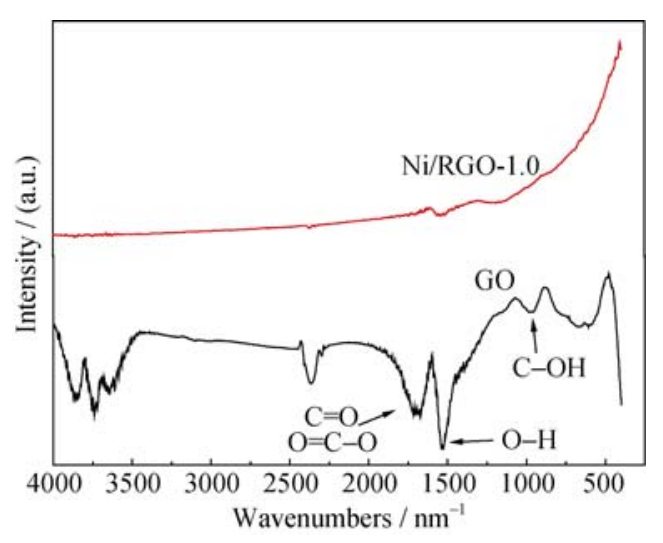

图 3 原始氧化石墨和 Ni/RGO-1.0 样品的红外光谱图谱

Fig. 3 FTIR spectra of GO and Ni/RGO-1.0

中的含氧官能团的各吸收振动峰在经过还原处理 后, 其强度明显减弱, 其中 $\mathrm{C}-\mathrm{OH}$ 的峰几乎完全消 失, 这说明经过热还原处理后，原材料层间的含氧 基团大部分已经去除, 复合材料得到了较高程度 的还原。

\section{$2.2 \mathrm{Ni} / \mathrm{RGO}$ 纳米复合材料的电磁参数分析}

Pinho 等 ${ }^{[20]}$ 在研究雷达吸波材料时提到, 在基 于传输/反射法模型的基础上, 微波吸波材料的相 对介电常数 $\varepsilon$ 与相对磁导率 $\mu$ 是表征其吸波性能的 重要参数。在交变磁场下, 两者常用复数形式表示 $\left(\varepsilon_{\mathrm{r}}=\varepsilon^{\prime}-j \varepsilon^{\prime \prime}\right.$ 与 $\left.\mu_{\mathrm{r}}=\mu^{\prime}-j \mu^{\prime \prime}\right)$, 其中相对复介电常数实部代 表材料储存能量的能力, 虚部表示对能量的损耗 能力; 相对复磁导率的实部与虚部则和磁介质内 储藏能量密度和磁损耗功率成正比。根据测得的相 对复介电常数和相对复磁导率的实部与虚部, 可 以利用式(1)计算出介电损耗角正切值 $\tan \delta_{\varepsilon}$ 和磁损 耗角正切值 $\tan \delta_{\mu}$ 。

$$
\tan \delta_{\varepsilon}=\varepsilon^{\prime \prime} / \varepsilon^{\prime} \quad \tan \delta_{\mu}=\mu^{\prime \prime} / \mu^{\prime}
$$

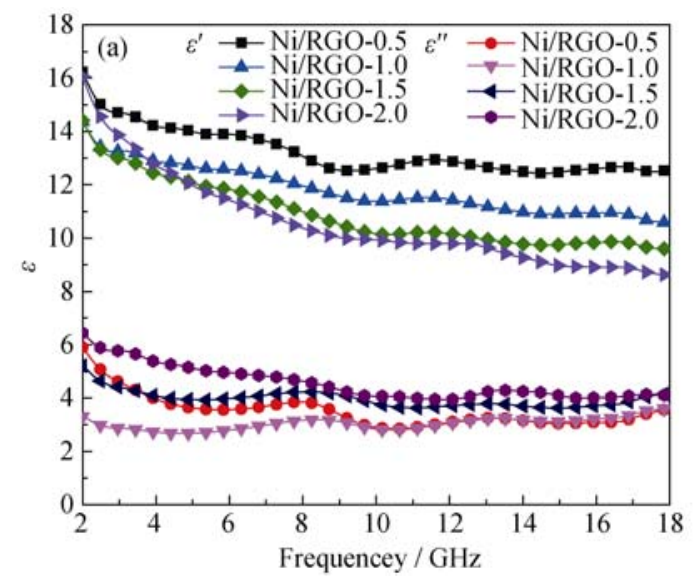

$\tan \delta_{\varepsilon}$ 和 $\tan \delta_{\mu}$ 经常被用来研究材料微波吸收性 能的内在原因以及机理, 通过分析其变化趋势和数 值大小，可推测出微波吸收过程中由尖端效应和极 化区域引起的电子自旋以及电荷极化对材料吸波性 能的影响 ${ }^{[21-23]}$ 。

图 4 列出了不同 $\mathrm{Ni}\left(\mathrm{NO}_{3}\right)_{2}$ 浓度下获得的 $\mathrm{Ni} / \mathrm{RGO}$ 纳米复合材料的电磁参数。由图 4(a)可知, 随着浓度的增加, 样品的 $\varepsilon^{\prime}$ 呈现逐渐减小的趋势。 根据上文对材料物相以及形貌的分析, 当浓度为 $0.5 \mathrm{~mol} / \mathrm{L}$ 时, 样品在测试频率范围内的平均 $\varepsilon^{\prime}$ 值约 为 14 , 而 $\mathrm{Ni} / \mathrm{RGO}-2.0$ 样品的则为 12 左右。而材料 的 $\varepsilon^{\prime \prime}$ 值从总体上来看却是随着浓度的增加而逐步上 升, 当 $\mathrm{Ni} / \mathrm{RGO}-0.5$ 样品在测试频率范围内的平均 $\varepsilon^{\prime \prime}$ 值大约为 4 时, $\mathrm{Ni} / \mathrm{RGO}-2.0$ 样品的平均 $\varepsilon^{\prime \prime}$ 值则上升 至 5 左右。该现象说明复合材料的导电性随着浓度 的提升而增强，分析其原因可能是随着浓度的增大， 得到还原生成的 $\mathrm{Ni}$ 颗粒也越多，而金属成分的增多 则增强了材料的导电性, 其电容性能则相应减弱, 所以造成 $\varepsilon^{\prime}$ 值的下降、 $\varepsilon^{\prime \prime}$ 值的升高, 这说明材料储存 电荷或者能量的能力有所下降，而对电磁波能量的 损耗能力有所增强。

由图 4(b)还可看出随着浓度的增加, 三个样 品的 $\mu^{\prime}$ 值只有轻微的降低, 推测与 $\mathrm{Ni}$ 含量的增加 有关, 而对于 $\mu^{\prime \prime}$ 值, 可以从图中发现, 在 $2 \sim 3 \mathrm{GHz}$ 这一低频区, Ni/RGO-0.5 样品的 $\mu^{\prime \prime}$ 值约为零, 而 随着 $\mathrm{Ni}\left(\mathrm{NO}_{3}\right)_{2}$ 浓度的提高, 该数值逐渐增大, $\mathrm{Ni} /$ RGO- 1.5 样品的 $\mu^{\prime \prime}$ 值高达 0.2 , 当浓度进一步上升至 $2.0 \mathrm{~mol} / \mathrm{L}$ 时, $\mu^{\prime \prime}$ 值又下降至 0.1 左右。分析其原因可 能是由于 $\mathrm{Ni}$ 金属为磁性材料, Ni/RGO-1.5 样品中 $\mathrm{Ni}$ 颗粒的数量较前两种样品要多, 同时晶粒的完整 度较前两者有所提高, 因此带来了磁性能的改

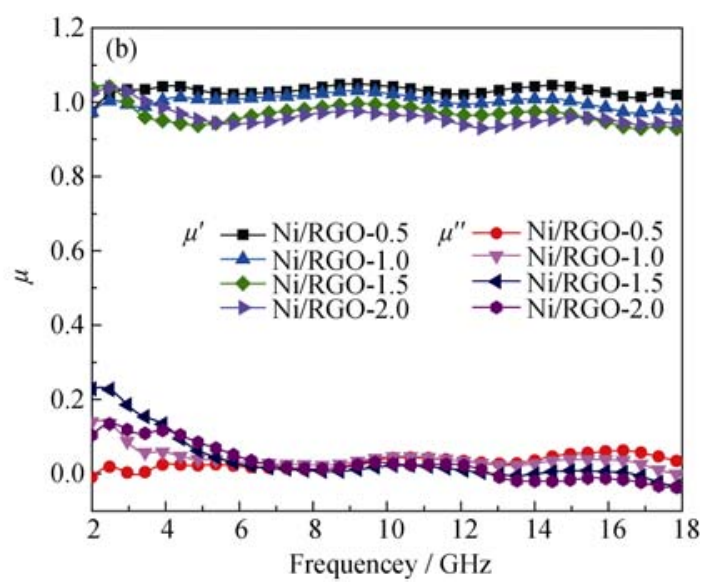

图 $4 \mathrm{Ni} / \mathrm{RGO}-0.5 、 \mathrm{Ni} / \mathrm{RGO}-1.0 、 \mathrm{Ni} / \mathrm{RGO}-1.5$ 和 $\mathrm{Ni} / \mathrm{RGO}-2.0$ 样品的电磁参数图谱

Fig. 4 Electromagnetic parameters of Ni/RGO-0.5, Ni/RGO-1.0, Ni/RGO-1.5, and Ni/RGO-2.0 
善 ${ }^{[24-25]}$, 但是当浓度进一步增大时, 由上文可知材 料此时导电性得到增强，致使材料与空气介质的对 应参数存在较大差异, 导致大部分的入射电磁波将 直接反射回空气中, 内部 $\mathrm{Ni}$ 无法得到全部利用, 所 以表现出 Ni/RGO-2.0 样品 $\mu^{\prime \prime}$ 值的下降。

图 5 是不同 $\mathrm{Ni}\left(\mathrm{NO}_{3}\right)_{2}$ 浓度下制得的 $\mathrm{Ni} / \mathrm{RGO}$ 纳 米复合材料 $\tan \delta_{\varepsilon}$ 和 $\tan \delta_{\mu}$ 与频率的关系曲线。由图 可知, 四个样品的介电损耗角的正切值 $\tan \delta_{\varepsilon}$ 在整个 $2 \sim 18 \mathrm{GHz}$ 频段内都大于磁损耗正切值 $\tan \delta_{\mu}$, 表明 $\mathrm{Ni} / \mathrm{RGO}$ 纳米复合材料是一种以电损耗为主的微波 吸收材料。而 Ni/RGO-1.5 样品的 $\tan \delta_{\mu}$ 在 2 6 GHz 频段要明显高于 $\mathrm{Ni} / \mathrm{RGO}-0.5$ 和 $\mathrm{Ni} / \mathrm{RGO}-1$, 即在这 个频段内，其磁损耗量有所提高。

利用所测得的各项电磁参数以及式(2)、(3)、(4), 可得出试样在不同厚度下的理论反射损耗(简称 $R L$ ) 随频率 $f$ 的变化曲线(图 5)。

$$
\begin{gathered}
\eta=\sqrt{\frac{\mu_{r}}{\varepsilon_{r}}} \operatorname{th}\left(j \frac{2 \pi d}{\lambda} \sqrt{\mu_{r} \varepsilon_{r}}\right) \\
R=(\eta+1) /(\eta-1) \\
R L(\mathrm{~dB})=201 \mathrm{~g}|R|
\end{gathered}
$$
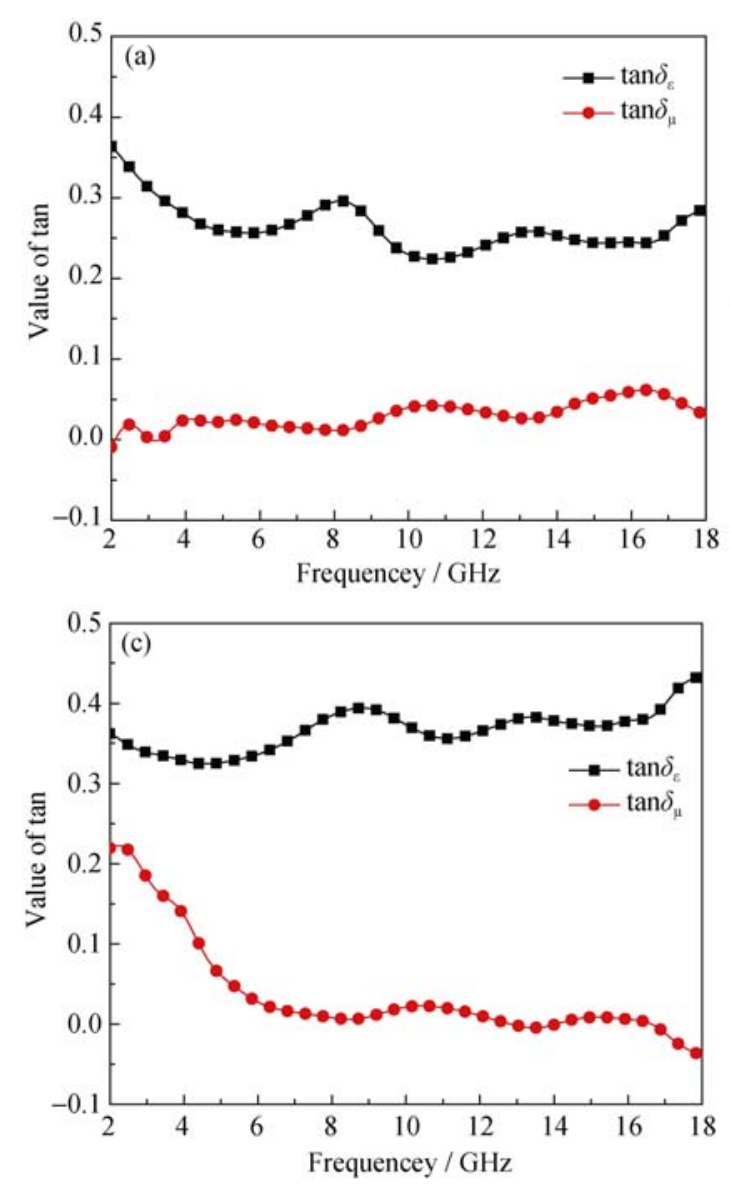

各样品理论损耗值随频率的变化如图 6 所示, 由图可知, 四个样品的 $R L$ 值随频率的变化大致相 似, 吸收频段均随着厚度的增加向低频方向移动。 但最大理论反射损耗 $R L_{\max }$ 值随着厚度的增加则有 所不同, 样品 Ni/RGO-0.5 呈增大趋势, 由-18.5 dB 减小至约 $-24 \mathrm{~dB}$, 其它三个样品呈减小趋势。三 个样品均在中低频段表现出了较好的吸波效果, 其中 $\mathrm{Ni} / \mathrm{RGO}-1.5$ 表现出最好的吸波性能, 当其厚 度为 $5 \mathrm{~mm}$ 时, 其 $R L$ 小于 $-5 \mathrm{~dB}$ 的匹配频段为 3.2 $6.0 \mathrm{GHz}$ ，带宽近 $3 \mathrm{GHz}$ 。

表 1 列出了所有样品在厚度为 $1.5 \mathrm{~mm}$ 和 $2 \mathrm{~mm}$ 时的主要电磁性能参数。由表可知, 当金属盐浓度 由 $0.5 \mathrm{~mol} / \mathrm{L}$ 增大至 $1.5 \mathrm{~mol} / \mathrm{L}$ 时, 复合材料的微波 吸收性能一直呈现增强的趋势, 其最大理论损耗 值可达 $-44 \mathrm{~dB}$ 。但是随着金属盐浓度的继续增长至 $2 \mathrm{~mol} / \mathrm{L}$ 时, 其性能反而有所下降。另外, $\mathrm{Ni} / \mathrm{RGO}-0.5$ 的 $R L_{\max }$ 的匹配频率偏低, 随着 $\mathrm{Ni}$ 含量的增加, $R L_{\max }$ 的匹配频率逐渐向高频方向移动。在厚度为 $1.5 \mathrm{~mm}$ 时, 样品 Ni/RGO-0.5 的 $R L$ 值小于 $-5 \mathrm{~dB}$ 的 频带宽度最大, 达到了 $6.5 \mathrm{GHz}$; 当厚度为 $2 \mathrm{~mm}$ 时,
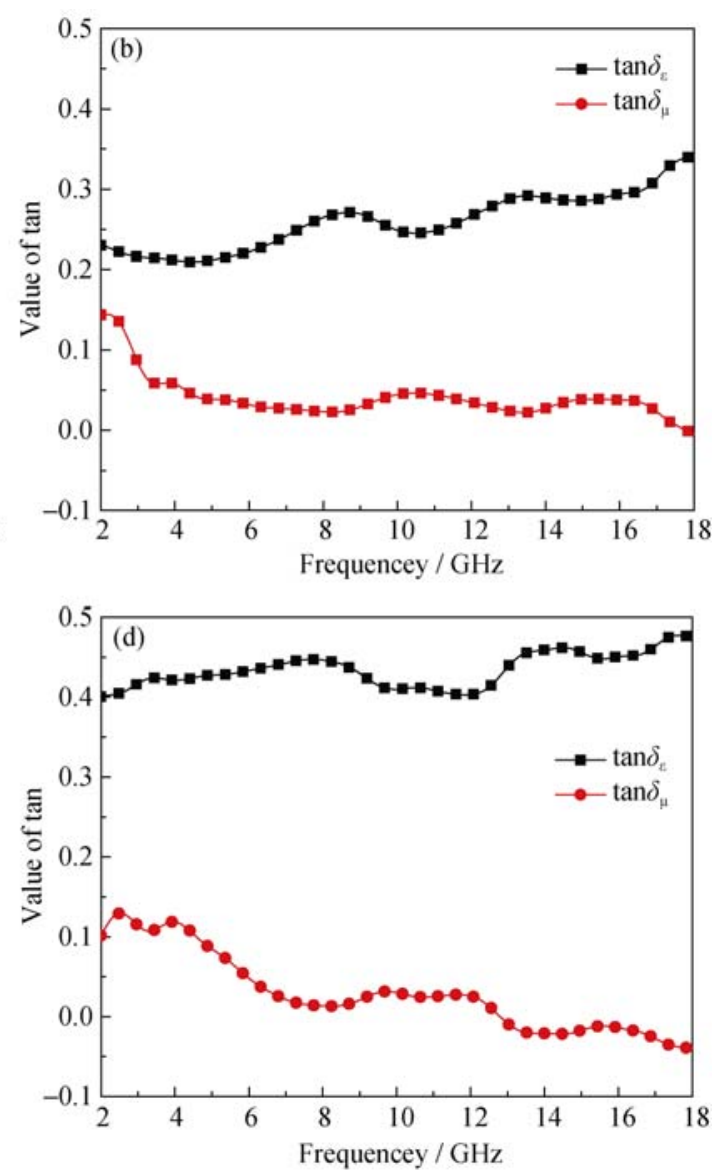

图 $5 \mathrm{Ni} / \mathrm{RGO}-0.5(\mathrm{a}) 、 \mathrm{Ni} / \mathrm{RGO}-1.0(\mathrm{~b}) 、 \mathrm{Ni} / \mathrm{RGO}-1.5(\mathrm{c})$ 和 Ni/RGO-2.0(d)样品的 $\tan \delta_{\varepsilon}$ 和 $\tan \delta_{\mu}$ 图谱

Fig. $5 \tan \delta_{\varepsilon}$ and $\tan \delta_{\mu}$ of Ni/RGO-0.5 (a), Ni/RGO-1.0 (b), Ni/RGO-1.5 (c), and Ni/RGO-2.0 (d) 

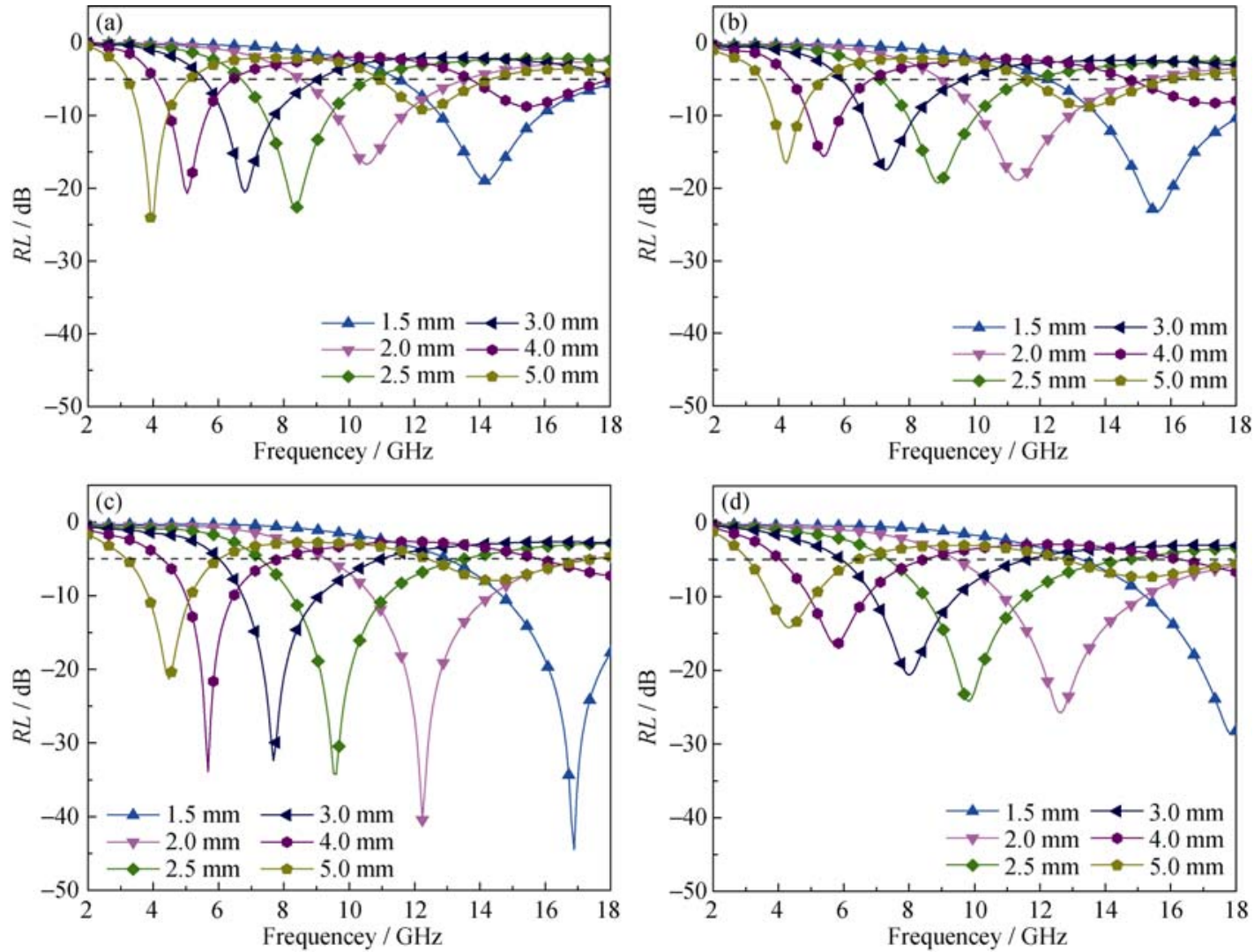

图 $6 \mathrm{Ni} / \mathrm{RGO}-0.5(\mathrm{a}) 、 \mathrm{Ni} / \mathrm{RGO}-1.0(\mathrm{~b}) 、 \mathrm{Ni} / \mathrm{RGO}-1.5(\mathrm{c})$ 和 $\mathrm{Ni} / \mathrm{RGO}-2.0(\mathrm{~d})$ 样品的理论反射损耗曲线

Fig. 6 Theoretical reflection losses of Ni/RGO-0.5 (a), Ni/RGO-1.0 (b), Ni/RGO-1.5 (c), and Ni/RGO-2.0 (d)

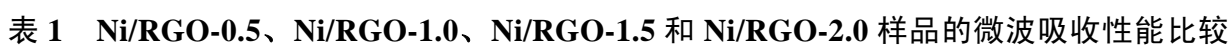

Table 1 Electromagnetic property of Ni/RGO-0.5, Ni/RGO-1.0, Ni/RGO-1.5, and Ni/RGO-2.0

\begin{tabular}{ccccccccc}
\hline Sample & \multicolumn{2}{c}{$\mathrm{Ni} / \mathrm{RGO}-0.5$} & \multicolumn{2}{c}{$\mathrm{Ni} / \mathrm{RGO}-1$} & \multicolumn{2}{c}{ Ni/RGO-1.5 } & \multicolumn{2}{c}{$\mathrm{Ni} / \mathrm{RGO}-2.0$} \\
\hline Material thickness $/ \mathrm{mm}$ & 1.50 & 2.0 & 1.5 & 2.0 & 1.5 & 2.0 & 1.5 & 2.0 \\
Absorbing scale lower than $-5 \mathrm{~dB} / \mathrm{GHz}$ & $11.5-18.0$ & $8.5-13.2$ & $12.4-18.0$ & $9.0-15.4$ & $13-18$ & $9-18$ & $13-18$ & $9.5-18.0$ \\
Absorbing width lower than $-5 \mathrm{~dB} / \mathrm{GHz}$ & 6.5 & 5.3 & 5.6 & 6.4 & 5.0 & 9.0 & 5.0 & 8.5 \\
Max value of $\mathrm{RL} / \mathrm{dB}$ & -18.5 & -17.0 & -23.0 & -18.0 & -44.0 & -40.0 & -28.8 & -25.9 \\
Matching frequence for $\mathrm{RL}_{\max } / \mathrm{GHz}$ & 14.0 & 10.5 & 15.5 & 11.2 & 17.0 & 12.2 & 17.8 & 12.6 \\
\hline
\end{tabular}

样品 $\mathrm{Ni} / \mathrm{RGO}-1.5$ 的频带宽度最大，达到了 $9 \mathrm{GHz}$, 覆盖了整个 $\mathrm{Ku}$ 波段和大部分 $\mathrm{X}$ 波段, 其 $R L_{\text {max }}$ 可达 $-40 \mathrm{~dB}$ 。对比四个样品的理论发射损耗曲线可知, 当吸收材料厚度较大时, $\mathrm{Ni} / \mathrm{RGO}-1.5$ 的吸收频段宽 度最大。以上性能表征说明, 在 $\mathrm{RGO}$ 和 $\mathrm{Ni}$ 之间存 在一定的复合效应, 能有效增强材料的微波吸收性 能。当 $\mathrm{Ni}$ 浓度较低, 金属颗粒尺寸较小时, 复合材 料的性能受 RGO 的影响较大, 从而表现出较好的 低频吸波特性。随着 $\mathrm{Ni}$ 含量的增加, 金属 $\mathrm{Ni}$ 在吸 波性能中的影响增强, 复合效应也有所增大, 对电 磁波的反射损耗能力明显增大, 微波吸收频段宽度 有了明显的拓展。但是其性能并非随着 $\mathrm{Ni}$ 含量的增 加而不断增强, 当浓度增加到一定程度时, 其吸波 性能反而会有所下降, 本研究中当金属盐浓度为
$1.5 \mathrm{~mol} / \mathrm{L}$ 时，材料表现出最佳吸波性能。

\section{3 结论}

以 $\mathrm{Ni}\left(\mathrm{NO}_{3}\right)_{2}$ 为 $\mathrm{Ni}$ 源, 利用液相吸附法在氧化 石墨层间吸附 $\mathrm{Ni}$ 离子并通过 $\mathrm{H}_{2}$ 热还原处理可获得 纳米 $\mathrm{Ni}$ 颗粒均匀附着在 $\mathrm{RGO}$ 表面的 $\mathrm{Ni} / \mathrm{RGO}$ 纳米 复合材料。系统研究了 $\mathrm{Ni}\left(\mathrm{NO}_{3}\right)_{2}$ 浓度对 $\mathrm{Ni} / \mathrm{RGO}$ 纳 米复合材料微结构以及微波吸收性能的影响。结果 表明:

1) 随着 $\mathrm{Ni}\left(\mathrm{NO}_{3}\right)_{2}$ 浓度增大, $\mathrm{Ni} / \mathrm{RGO}$ 纳米复合材 料中 $\mathrm{Ni}$ 颗粒数量逐渐增多, 其颗粒尺寸也随之增大。

2) 电磁参数测试结果表明, 当 $\mathrm{Ni}\left(\mathrm{NO}_{3}\right)_{2}$ 浓度 增大到 $1.5 \mathrm{~mol} / \mathrm{L}$ 时, 材料的相对复磁导率虚部 $\mu^{\prime \prime}$ 
值在 $2 \sim 6 \mathrm{GHz}$ 低频区内略有上升，材料磁损耗有 增强趋势。

\section{3) 理论反射损耗计算表明, Ni/RGO 纳米复合材} 料是一种良好的微波吸收材料, 其中 Ni/RGO-1.5 性 能最好, 样品厚度为 $2 \mathrm{~mm}$ 时, 其 $R L$ 小于 $-5 \mathrm{~dB}$ 的吸 收频带宽度可达 $9 \mathrm{GHz}, R L_{\max }$ 可达 $-40 \mathrm{~dB}$; 在低频区 亦能保持较好的微波吸收效果。

\section{参考文献:}

[1] SHI H Y, LI P, MA Y, et al. The high-power electromagnetic radiation hazard to human body and protective measures. New Technology \& New Products of China, 2010, 23: 13.

[2] DIAO Q A, GAO G G. The ecological damage caused by electromagnetic environment and protection. Basic \& Clinical Medicine, 2000, 20(1): 3-7.

[3] YE W C, FU J J, WANG Q, et al. Electromagnetic wave absorption properties of $\mathrm{NiCoP}$ alloy nanoparticles decorated on reduced graphene oxide nanosheets. Journal of Magnetism and Magnetic Materials, 2015, 395: 147-151.

[4] WANG G, CHANG Y, WANG L, et al. Synthesis, characterization and microwave absorption properties of $\mathrm{Fe}_{3} \mathrm{O}_{4} / \mathrm{Co}$ core/shell-type nanoparticles. Advanced Powder Technology, 2012, 23(6): 861-865.

[5] ZHANG T, HUANG D, YANG Y, et al. $\mathrm{Fe}_{3} \mathrm{O}_{4} /$ carbon composite nanofiber absorber with enhanced microwave absorption performance. Materials Science and Engineering B, 2013, 178(1): 1-9.

[6] JIA K, ZHAO R, ZHONG J, et al. Preparation and microwave absorption properties of loose nanoscale $\mathrm{Fe}_{3} \mathrm{O}_{4}$ spheres. Journal of Magnetism \& Magnetic Materials, 2010, 322(15): 2167-2171.

[7] ZHANG X C, ZHAO Z S, HE H H, et al. Temperature character of complex permeability and permittivity of microwave ferrite absorbent. Journal of Functional Materials, 1994, 25(2): 169-171.

[8] 刘 归. 纳米 $\mathrm{Fe}_{3} \mathrm{O}_{4}$ 复合材料的微波吸收特性研究. 湖南长沙: 中南大学硕士学位论文, 2005.

[9] ZHAO B, FAN B, XIE Y, et al. Effect of particle sizes on the microwave absorption properties of monodispersed $\mathrm{Ni}$ submicrospheres. Optik, 2015, 126(23): 4597-4600.

[10] WU N, LIU X, ZHAO C, et al. Effects of particle size on the magnetic and microwave absorption properties of carbon-coated nickel nanocapsules. Journal of Alloys \& Compounds, 2016, 656: 628-634.

[11] WANG C, KANG F Y, GU J L, et al. Synthesis and microwave absorbing properties of $\mathrm{Fe}-\mathrm{Co}-\mathrm{Ni}$ alloy particles/graphite flaky composite. Journal of Inorganic Materials, 2010, 25(4): 406-410.

[12] ZOU Y H, LIU H B, YANG L, et al. Influence of reduction temperature on structure and microwave absorption property of
Fe/Graphite nanocomposite. Materials Review, 2007, 21(11): 144-148.

[13] XU C X, YANG W B, XIE C Q, et al. Preparation and microwave absorbing property of electroless plating Ni-Co-P alloy on surface of flake graphite. Journal of Southwest University of Science and Technology, 2013, 28(1): 13-16.

[14] ZHU M Y, DIAO G W. Review on the progress in synthesis and application of magnetic carbon nanocomposites. Nanoscale, 2011, 3(7): 2748-2767.

[15] LI J, LIU H B, YANG L. Research on Microwave absorption properties of $\mathrm{FeCo} /$ Graphite nanocomposite. Journal of Inorganic Materials, 2014, 29(5): 470-474.

[16] YANG LI, LIU H B, LI F J, et al. Characterization and microwave absorption properties of ternary $\mathrm{FeCl}_{3}-\mathrm{NiCl}_{2}$-GICs. New Carbon Materials, 2008, 23(4): 367-373.

[17] MENG H J, ZHAO X W, YU L G, et al. Island-like nickel/carbon nanocomposites as potential microwave absorbers-Synthesis via in situ solid phase route and investigation of electromagnetic properties. Journal of Alloys and Compounds, 2015, 644: 236-241.

[18] SUN D, ZOU Q, WANG Y, et al. Controllable synthesis of porous $\mathrm{Fe}_{3} \mathrm{O}_{4} @ \mathrm{ZnO}$ sphere decorated graphene for extraordinary electromagnetic wave absorption. Nanoscale, 2014, 6(12): 6557-6562.

[19] YANG X, CHEN C L, LI J X, et al. Graphene oxide-iron oxide and reduced graphene oxide-iron oxide hybrid materials for the removal of organic and inorganic pollutants. Journal of RSC Advances. 2012, 2(23): 8821-8826.

[20] PINHO M S, GREGORI M L, REIS N R C, et al. Performance of radar absorbing materials by waveguide measurements for $\mathrm{X}$ - and Ku-band frequencies. European Polymer Journal, 2002, 38(11): 2321-2327.

[21] YANG Y, GUPTA M, DUDLEY K, et al. Novel carbon nanotube-polystyrene foam composites for electromagnetic interference shielding. Nano Lett, 2005, 5(11): 2131-2134.

[22] MANDAL S, SAHA S. Ni/graphene/Ni nanostructures for spintronic applications. Nanoscale, 2012, 4(3): 986-990.

[23] CHEN T T, DENG F, ZHU J, et al. Hexagonal and cubic Ni nanocrystals grown on graphene: phase-controlled synthesis, characterization and their enhanced microwave absorption properties. J. Mater. Chem., 2012, 22(30): 15190-15197.

[24] ANDREEV V G, MENSHOVA S B, KLIMOV A N, et al. The influence of basic composition and microstructures on the properties of $\mathrm{Ni}-\mathrm{Zn}$ ferrite radio-absorbing materials. Journal of Magnetism and Magnetic Materials, 2015, 393: 569-573.

[25] WANG T S, LIU Z H, LU M M, et al. Graphene-- $\mathrm{Fe}_{3} \mathrm{O}_{4}$ nanohybrids: synthesis and excellent electromagnetic absorption properties. Journal of Applied Physics. 2013, 113(2): 024314-1-8. 